\title{
DNA meets synthetic polymers—-highly versatile hybrid materials
}

\author{
Fikri E. Alemdaroglu and Andreas Herrmann*† \\ Received 8th December 2006, Accepted 6th February 2007 \\ First published as an Advance Article on the web 21st February 2007 \\ DOI: 10.1039/b617941j
}

The combination of synthetic polymers and DNA has provided biologists, chemists and materials scientists with a fascinating new hybrid material. The challenges in preparing these molecular chimeras were overcome by different synthetic strategies that rely on coupling the nucleic acid moiety and the organic polymer in solution or on solid supports. The morphologies and functions of the bioorganic block copolymers can be controlled by the nature of the synthetic polymer segment as well as by the sequence composition and length of the DNA. Recent developments have expanded the scope and applications of these hybrid materials in a number of different areas including biology and medicine, as well as bio- and nanotechnology. Their usage ranges from gene delivery through to DNA detection to programmable nano-containers for DNA-templated organic reactions.

\section{Introduction}

Hybrids are a combination of dissimilar components arranged at the nanometric and molecular level., ${ }^{1,2}$ Throughout evolution, nature has evolved a large variety of hybrid materials, if one thinks of the post-transcriptional modifications of proteins, where peptidic structures are functionalized with carbohydrates or lipids, ${ }^{3}$ and the process of biomineralization, ${ }^{4,5}$ which combines organic and inorganic materials within biological systems. Natural hybrids containing nucleic acids as a major class of biomacromolecules are also known. One important example is the ribosome which consists of an RNA structure into which

Max Planck Institute for Polymer Research, Ackermannweg 10, 55128, Mainz, Germany. E-mail: andherrm@mpip-mainz.mpg.de; Fax: +496131379100; Tel: +49 6131-379336

$\dagger$ Homepage : http://www.mpip-mainz.mpg.de/ andherrm proteins are interdispersed by non-covalent bonds. ${ }^{6}$ Especially the complex function of this entity, i.e. the catalysis of protein biosynthesis, underlines the importance and potency of such biological hybrids. Involved in this process is another type of molecular chimera, the so-called tRNAs. They consist of RNA covalently linked to small organic molecules, the amino acids. ${ }^{7}$ Beside these naturally occurring examples, chemists have created artificial nucleic acid hybrid structures. DNA has been combined with inorganic materials like gold nanoparticles but also with small organic moieties like organic dyes or electrochemically active units. ${ }^{8,9}$ With such DNA hybrids, new detection strategies ${ }^{10}$ and nanoelectronic structures, ${ }^{11,12}$ as well as nanomechanical devices, ${ }^{13}$ have been realized. In recent years a new type of nucleic acid hybrid has emerged, which consists of the combination of synthetic oligonucleotides (ODNs) and organic polymers. As a consequence of joining these two classes of materials, DNA block copolymers (DBCs) are formed that maintain the special features

Fikri E. Alemdaroglu was born in Adana (Turkey) in 1979 and obtained his B.Sc. in chemistry at Bogazici University (Turkey). After graduation, he moved to the Free University of Berlin (Germany) and received his M.Sc. in Polymer Science with a master thesis in the group of Professor A. D. Schlüter on the synthesis of amphiphilic poly(para-phenylenes). Since 2004, he has been at the Max Planck Institute for Polymer Research (Germany) working on his Ph.D. under the supervision of Dr A. Herrmann and Professor K. Müllen. The topic of his research consists of the synthesis, self-assembly and applications of DNA block copolymers.

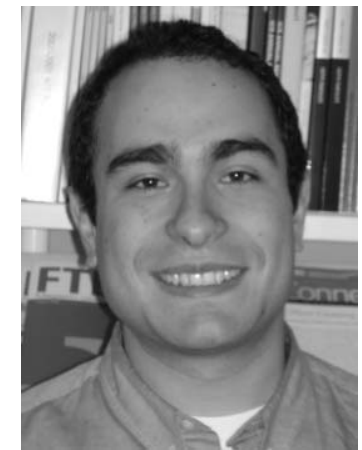

Fikri E. Alemdaroglu

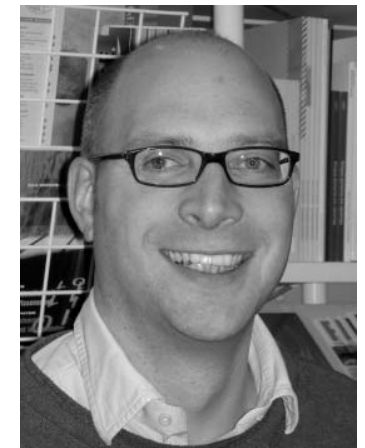

Andreas Herrmann
Andreas Herrmann studied chemistry at the University of Mainz (Germany). From 1997 to 2000 he pursued his graduate studies at the Max Planck Institute for Polymer Research in the group of Professor K. Müllen. He then worked as a consultant for Roland Berger Management Consultants in Munich (2001). In 2002 and 2003 he returned to academia, working on protein engineering at the Swiss Federal Institute of Technology, Zurich, with Professor D. Hilvert. In 2004 he was appointed as a head of a junior research group at the Max Planck Institute for Polymer Research dealing with biological-organic and biological-polymeric hybrid materials. The current research interests of the group are twofold: 1) combining synthetic strategies from organic and polymer chemistry with methods from molecular biology; and 2) exploring the interactions of bioorganic hybrid materials with living systems. 
of the biomacromolecule DNA and at the same time represent polymeric block type architectures that have attractive material properties in their own right.

The special features of DNA that are important in regard to the corresponding polymeric hybrids are the following: 1) Solidphase organic synthesis methods allow the preparation of singlestranded (ss) DNA with almost any desired sequence of more than 100 bases. ${ }^{14}$ 2) Hybridization of complementary sequences leads to the formation of a helical, semiflexible double-stranded (ds) polymer with a diameter of about two nanometres and a pitch of about 3.4-3.6 $\mathrm{nm}$ in the B-form. 3) In addition to the famous double helix, ${ }^{15,16}$ DNA can adopt other superstructures such as triple helices, quadruplexes and sophisticated artificially created 2D and 3D nanostructures. ${ }^{17-20}$ 4) Finally, enzymes allow site specific modifications of the DNA strands.

In contrast, synthetic block copolymers usually self-assemble into well-ordered periodic structures, a phenomenon called microphase separation. ${ }^{21}$ This process is driven by the enthalpy of demixing of the constituent components of the block copolymers, whilst the macroscopic separation is hindered by the connectivity of the two blocks. Hence, the domain size of the ordered structures is of similar magnitude to that of the molecular dimensions. The morphologies which are adopted range from spherical, through cylindrical and gyroidal, to lamellar structures, and can be controlled by the block length ratio of the constituent components. Besides the formation of nanostructures in bulk, block copolymers also form nano-objects in solution. This is the case when one of the blocks dissolves in the solvent, while the other block is insoluble (selective solvents). Polyelectrolyte block copolymers, which combine structural features of polyelectrolytes, block copolymers, and surfactants, in particular show a rich association behaviour. The formation of micelles, strings, and networks of sometimes quite complicated topology has been described. ${ }^{22}$ This class of polymers is important to mention in the context of DNA block copolymers since DNA from a polymer chemist's point of view represents a polyelectrolyte.

DNA and synthetic polymers were combined to bring out or enhance advantageous chemical and biological behaviours and at the same time to reduce or wholly suppress undesirable properties. An additional target is the evolution of entirely new material behaviours.

In this review, we first describe the different routes to prepare DNA block copolymers. Special attention will be paid to the synthesis of linear topologies and graft architectures where ODNs are attached as side chains to a synthetic polymer backbone. Common to all of these structures is that the nucleic acid segments and the organic polymer moieties are connected by covalent bonds. There is a considerable amount of literature describing electrostatic complexes of DNA with various polycations, ${ }^{23,24}$ but this is beyond the scope of this review. In the second part we focus on the properties of these materials and discuss their applications in the fields of biology, biotechnology and nanoscience. Finally, some of the remaining challenges of these new bioorganic hybrid materials are highlighted.

\section{Synthesis of DNA block copolymers}

For the generation of linear DNA block copolymers, one end of an ODN needs to be coupled to a terminal functionality of an organic polymer block. This synthetic goal is achieved by grafting strategies, either by connecting the biological and the organic polymer segments in solution (Fig. 1A) or on a solid

A

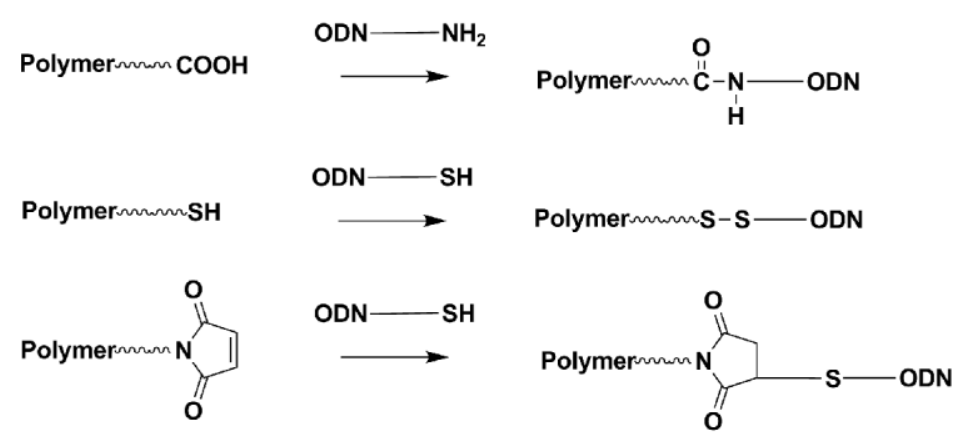

B
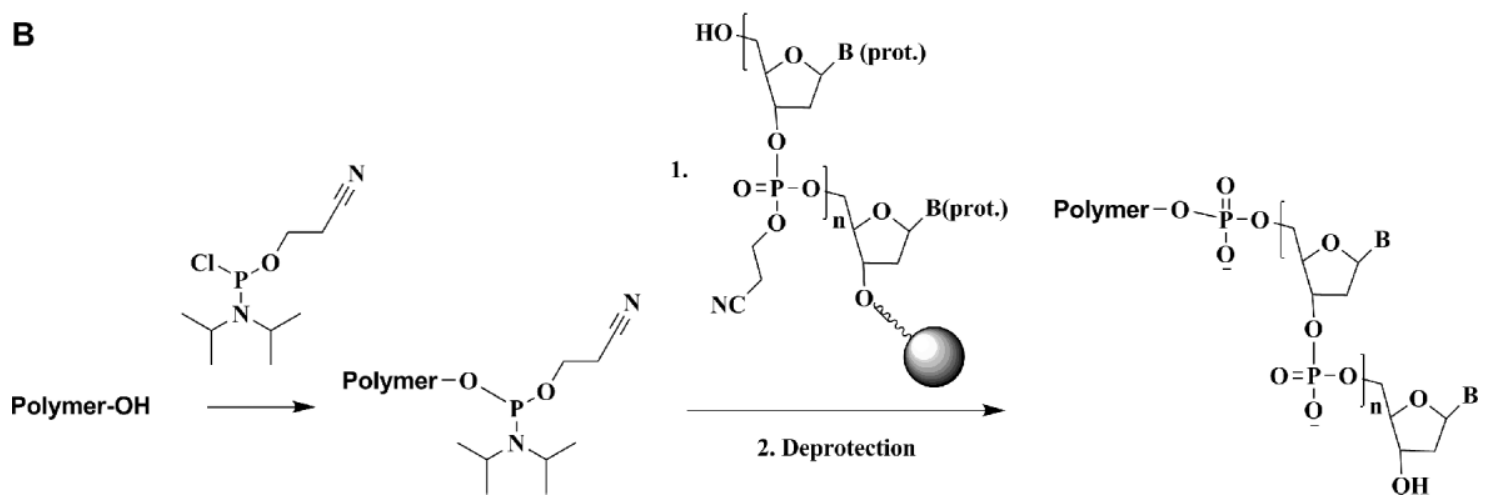

Fig. 1 The synthesis of linear DBCs by grafting onto in solution (A) and on the solid support (B). 
support (Fig. 1B). Three different coupling reactions in solution have been reported: amide ${ }^{25-28}$ and disulfide bond formation ${ }^{29}$ as well as Michael addition. ${ }^{29}$ When a peptide bond is formed to join both segments, terminally amino-functionalized ODNs were coupled to active ester-containing polymers. Several activating reagents including $N, N^{\prime}$-dicyclohexylcarbodiimide (DCC), $N$ hydroxysuccinimide ester (NHS) or $N$-(3-dimethylaminopropyl)$N^{\prime}$-ethylcarbodiimide (EDC) and sulfo- $N$-hydroxysuccinimide ester (sulfo-NHS) were used for the coupling reaction. The formation of a disulfide bridge between DNA and the polymer required a terminal thiol-modification at the ODN as well as at the polymer, which were reacted under slightly alkaline conditions in an aqueous phase. In the case of the Michael addition, thiol-functionalized ODNs were reacted with a malimido-functionalized polymer at neutral $\mathrm{pH}$. Attaching the biological and organic segments in solution is an easy procedure and does not require an expensive DNA synthesizer. Amino- or thiol-functionalized ODNs are available from commercial sources, which makes DBCs available to conventionally equipped laboratories. This coupling strategy proceeds with high yields as long as water-soluble polymers such as poly(ethylene oxide) (PEO) and poly ( $N$-isopropylacrylamide) (PNIPAM) are employed. ${ }^{28,30-38}$ However, the yields are drastically lower when hydrophobic polymers are used. A reason for poor coupling efficiencies is the incompatibility of the hydrophilic DNA and the hydrophobic polymers in the solvent. To overcome these synthetic difficulties, solid-phase synthesis was employed successfully for the preparation of amphiphilic DBCs by several groups, including ours (See Fig. 1B). ${ }^{39-43}$ The grafting approach on the solid support started with hydroxyl-terminated polymers that were reacted with phosphoramidite chloride to yield the corresponding phosphoramidite-polymer derivatives. This key reagent was then coupled to the detritylated 5' hydroxyl-end of the ODN on the solid support using a DNA-synthesizer. After liberation from the solid support, removal of the protecting groups and purification by polyacrylamide gel electrophoresis (PAGE) or by HPLC the DBCs were obtained in high yields. This synthetic route offers two advantages in regard to the preparation of amphiphilic DBCs. First, the incompatibility of the biological and the synthetic moiety is avoided because the coupling step is carried out in organic solvents in which the organic polymer is readily soluble. Second, the amphiphilic DBCs are produced fully automated in a single process. This is possible because phosphoramidite chemistry was employed for both the building of the ODN as well as for the coupling reaction of the polymer. Alternatively, the coupling of the organic polymer to the ODN can be carried out manually by the so-called "syringe synthesis technique" ${ }^{44,45}$ The latter method might have some drawbacks because high reproducibility and efficient exposure of the phosphoramidite polymer to the solid phase is not guaranteed to the same extent as in a DNA synthesizer.

Another structurally important class of DBCs consists of graft architectures where several ODNs are attached to the polymer backbone to form a comb-like topology (Fig. 2). Three different synthetic routes were developed to realize these structures. In the first approach, the synthetic polymer was prefabricated, and in a subsequent grafting step the ODNs were coupled in solution. One way to attach the ODNs to the synthetic backbone is amide bond formation. Therefore, during the synthesis the polymer backbone was equipped with active ester groups that were reacted with terminal amino-modified ODNs. ${ }^{46}$ As in the previous procedure, a covalent bond between the organic polymer and the nucleic acid units was realized with the help of amino-modified ODNs. They were reacted with an alternating copolymer consisting of ethylene and maleic anhydride units representing the backbone. ${ }^{47}$ A second route for the preparation of graft architectures relies on coupling the synthetic polymer to the ODN on a solid support. This procedure is similar to the one described above for the fabrication of linear amphiphilic DBCs using phosphoramidite polymers. A major difference was that several phosphoramidite groups along the polynorbornene backbone served as attachment points for the ODNs. ${ }^{44,45}$ A third variant for the preparation of DNA side chain polymers is based on polymerizable

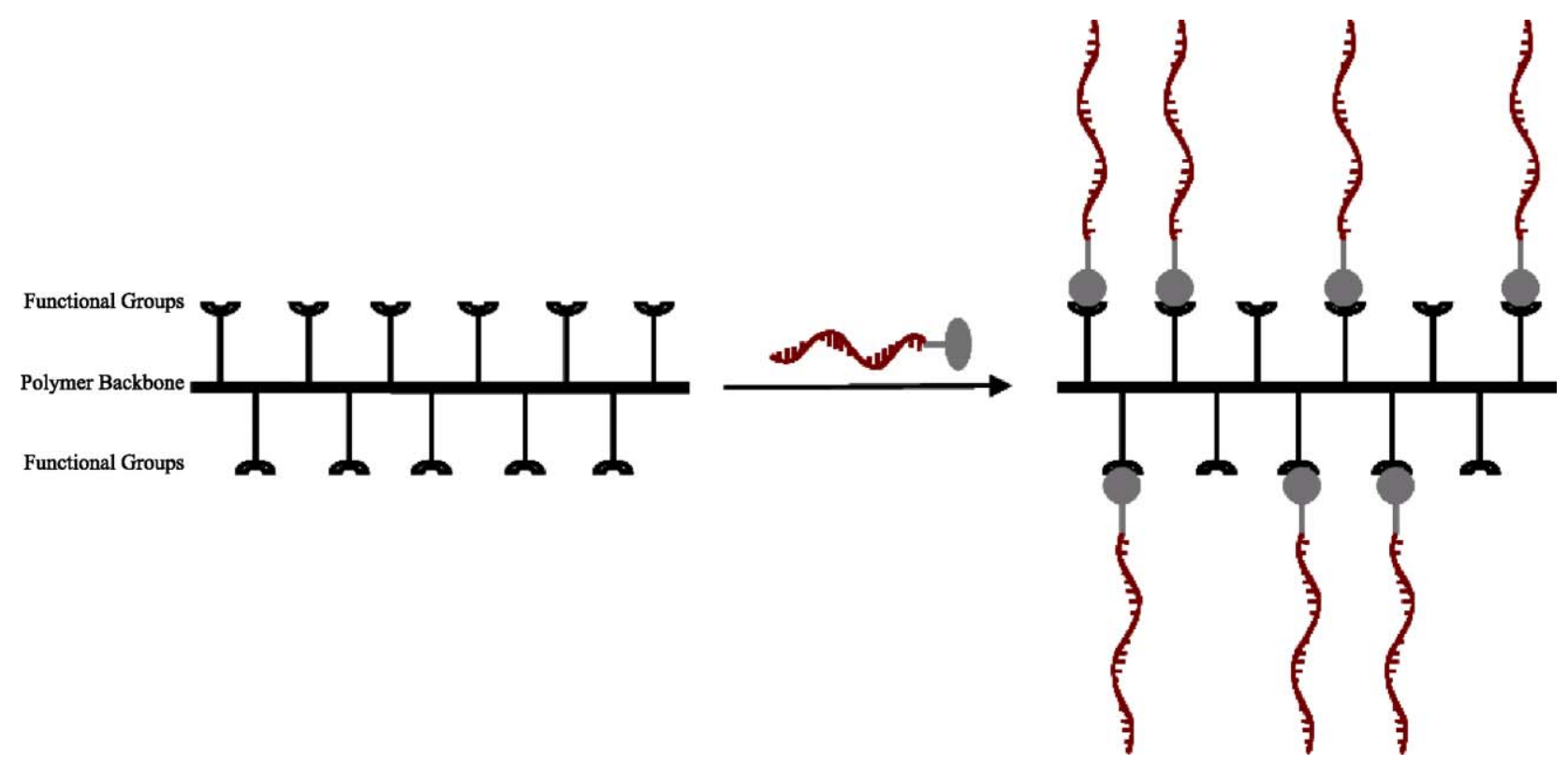

Fig. 2 The covalent attachment of terminally functionalized ODNs to a synthetic polymer backbone. 
ODN-macromonomers. An acrylamide monomer was functionalized via an alkyl spacer with a phosphoramidite group that can be reacted with the 5'-end of an ODN. This polymerizable nucleic acid moiety was transformed into a graft architecture by copolymerization with acrylamide. ${ }^{48,49}$ The multimerization of nucleic acid segments along a single organic macromolecule offers important advantages in some applications, including DNA detection and DNA hydrogels, which are discussed later in this review.

\section{DBCs as a new gene delivery system}

A wide variety of antisense oligonucleotides (ASOs) have attracted considerable attention due to their specific interaction with cytoplasmic mRNA, and therefore the blocking of specific gene products. ASOs are not only a useful experimental tool in protein target identification and validation for drug development, but also a highly selective therapeutic strategy for diseases with dysregulated protein expression. ${ }^{50}$ Practical applications of ASOs as therapeutic agents encounter two important problems: poor cellular uptake and enzymatic hydrolysis. ${ }^{51}$ This is the point where DBCs come into play because cellular uptake of ODNs can be enhanced and nuclease activity on ODN substrates can be reduced.

Park and co-workers have addressed the issue of poor cellular uptake by employing micellar aggregates of different DBCs as ASOs delivery systems. They prepared a DNA- $b$-poly(D,L-lacticco-glycolic acid) (PLGA) block copolymer by reacting amineterminated ASO with an activated PLGA. This amphiphilic DBC formed micelles readily in aqueous solution with PLGA segments as a hydrophobic core and ODN segments as a surrounding hydrophilic corona. Atomic force microscopy (AFM) and dynamic light scattering (DLS) analysis revealed spherical micelles with a diameter of $80 \mathrm{~nm}$. The in vitro uptake studies with NIH3T3 mouse fibroblast cells showed that the micelles were transported into the cells more efficiently than the pristine ODN. Due to the biodegradable nature of the organic polymer, these micelles could release the ASO in a controlled manner. ${ }^{52}$ The use of micelles as ASO carriers encouraged the same group to extend their delivery system to biocompatible DNA- $b$-PEO block copolymer systems. In this case, nanoscopic aggregates were prepared by complexation with polycations such as the positively charged fusogenic peptide KALA,${ }^{53}$ and polyethyleneimine (PEI) ${ }^{30}$ Both electrostatic complexes exhibited a core containing the charged moieties, whereas the corona was composed of PEO. The effective hydrodynamic diameter of both micelle aggregates was around $70 \mathrm{~nm}$, with a very narrow size distribution. In the first conjugate, the ODN was coupled to PEO via an acid-cleavable linkage (phosphoramidate) so that the ODN could be released in the acidic endosomal environment and could interact with the target mRNA sequence to inhibit protein expression. In particular, the cellular uptake behaviour and antiproliferation effects of the $c-m y b$ antisense ODN-containing polyion complex micelles on smooth muscle cells were investigated. It was shown that the micelles were incorporated into the cells far more efficiently than the non-polymer-modified ODN. Alternatively, the PEI cationic polymer was complexed with DNA- $b$-PEO that codes for $c$-raf antisense, and the corresponding electrostatic aggregate was applied to tumour-bearing nude mice. Significant antitumour activities against human lung cancer were measured. Interestingly, the polyion complex micelles showed a higher accumulation level in the tumour cells than the pristine
ODN. Kataoka et al. have synthesized electrostatic complexes of DNA- $b$-PEO and polycationic moieties such as PEI and poly(L-lysine) (PLL). ${ }^{33,38}$ The micelle systems containing PEI were designed in such a fashion that the ODN can be released by hydrolysis from the PEO segment. Moreover, the stability of the DNA- $b$-PEO within the polyion complex micelles against deoxyribonuclease (DNase I) was demonstrated. Important findings in regard to design effective antisense ODN delivery systems were made with the electrostatically trapped micelles bearing PLL as the polycation. Structural features of the DNA block copolymer also included an acid labile linker between the PEO and the nucleic acid moiety, and a lactose-targeting moiety attached to the PEO segment. A significant antisense effect against luciferase gene expression was observed. Micelles with a targeting unit showed a more pronounced antisense effect than control complexes without the lactose unit. The acid-labile linkage was found to be crucial for high antisense activity, since control experiments with a non-cleavable control DNA block copolymer showed decreased performance.

Besides targeting mRNA, recently so-called antigene ODNs that interact with ds DNA have been developed. These ODNs are designed to bind to polypurine-polypyrimidine sequences through triple helix formation and, thus manipulate gene function. ${ }^{54-62} \mathrm{~A}$ comprehensive study using DNA- $b$-PEO conjugates as antigene ODN delivery systems for inhibiting the expression of the Ki-ras gene and the proliferation of pancreatic cancer cells was carried out by Xodo and co-workers. ${ }^{36} \mathrm{~A}$ high molecular weight PEO was conjugated to a G-rich oligonucleotide as previously reported by the same group. ${ }^{63,64}$ The uptake of DNA- $b$-PEO, which was supposed to form a triplex with the promoter region of the $\mathrm{Ki}$ ras gene, was investigated by FACS and confocal fluorescence microscopy, showing that the cells harboured the conjugate at a concentration 6-7 times higher than the pristine ODN (Fig. 3). Of equal importance is that the DNA- $b$-PEO efficiently inhibited the transcription of Ki-ras mRNA, and the proliferation of pancreatic cancer cells was reduced by $50 \%$. It is important to mention that the ODN-PEO conjugate itself did not promote any inhibition of transcription by the anticipated interaction with the ds DNA. Instead, the antiproliferative activity was induced by binding of the DNA- $b$-PEO to a nuclear factor recognizing the Ki-ras promotor sequence by an aptameric mechanism. In this regard, the study introduced a new antiproliferative strategy based on the use of aptamers against nuclear proteins. On the other hand, this was the first report of an aptamer consisting of a DNA block copolymer.

\section{DBCs used in purification of biomaterials}

An important class of DBCs consists of the DNA- $b$-PNIPAM conjugates, which are used for purification of biomacromolecules employing a thermal stimulus. It is well-known that PNIPAM exhibits a remarkable phase transition in aqueous media in response to changes in temperature and therefore exhibiting a lower critical solution temperature (LCST). ${ }^{65,66}$ This fully reversible temperature-responsive behaviour has found application in the purification of bioconjugates from reactants and other solutes employing small temperature increases above the LCST. $^{26-28,31,32,67-72}$ In an important report, Freitag and co-workers synthesized a DNA- $b$-PNIPAM conjugate, of which the nucleic acid segment was capable of recognizing a sequence of plasmid DNA by triple 
a

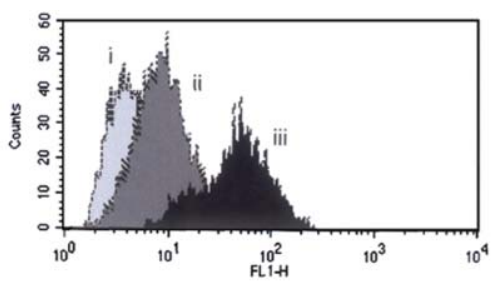

b

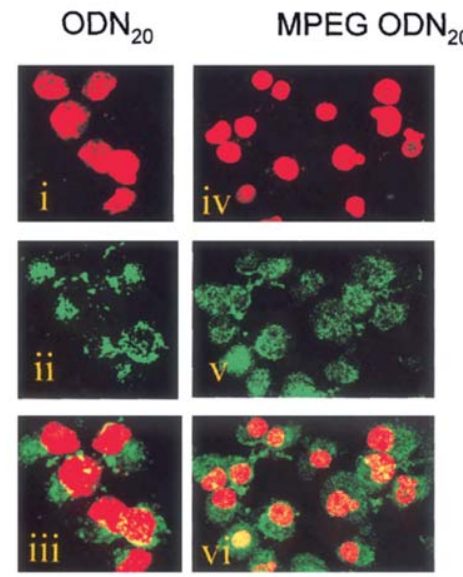

c
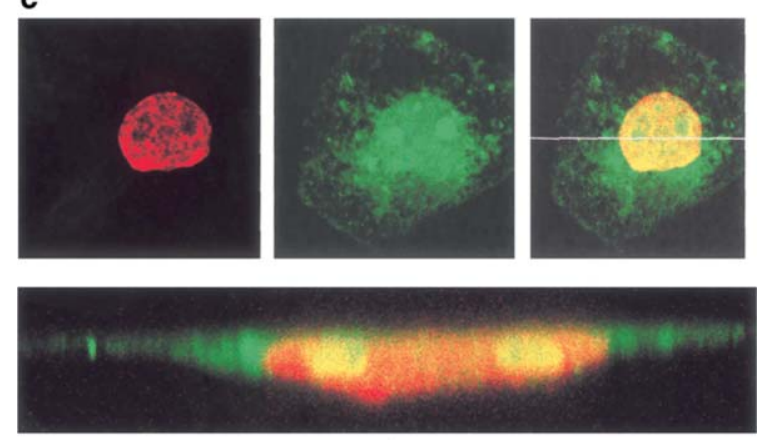

$\mathrm{x}-\mathrm{z}$ section

Fig. 3 Cellular uptake of pristine ODNs (20-mer) and DBCs consisting of a PEO and a nucleic acid block (20-mer). (a) FACS analysis of Panc-1 cells untreated and treated with $5 \mu \mathrm{M}$ ODN and MPEO- $b$-ODN. Cells were analyzed by FACS 48 hours after the oligonucleotides were delivered to the cells. Peak i, untreated cells; peak ii, cells treated with ODN-fluorescein (F); peak iii, cells treated with MPEO-b-ODN-F. (b) Confocal images of Panc- 1 cells treated for 24 hours with $5 \mu \mathrm{M}$ ODN-F (panels i, ii, iii) and MPEO-ODN20-F (panels iv, v,vi). Panels i and iv show the nuclei of Panc-1 cells stained in red with propidium iodide; panels ii and $\mathrm{v}$ show the green fluorescence emitted by the fluorescein-conjugated oligonucleotides; panels iii and vi are superimposed views obtained from $\mathrm{i}+$ ii and iv + v. (c) Confocal views of a Panc-1 cell showing that MPEO- $b-O D N 20-F$ is harboured in the nucleus. Note the presence of the conjugate in the nucleoli. The $x-z$ panel shows a cumulative projection of $x-z$ cross-sections corresponding to the line depicted in the magnified cell. Reprinted with permission from Nature Publishing Group (ref. 36).

helix formation (Fig. 4). After complexation below the LCST, the plasmid target DNA could be precipitated quantitatively from the solution by raising the temperature to $40{ }^{\circ} \mathrm{C}$. After redissolution at lower temperatures, DNA-b-PNIPAM was released from the plasmid by changing the $\mathrm{pH}$ of the solution. The target DNA molecule was obtained in yields of 70 to $90 \%$ in good purity. ${ }^{71}$ Plasmid DNA offers an attractive way to deliver therapeutic genes for gene therapy and genetic immunization due to its simplicity
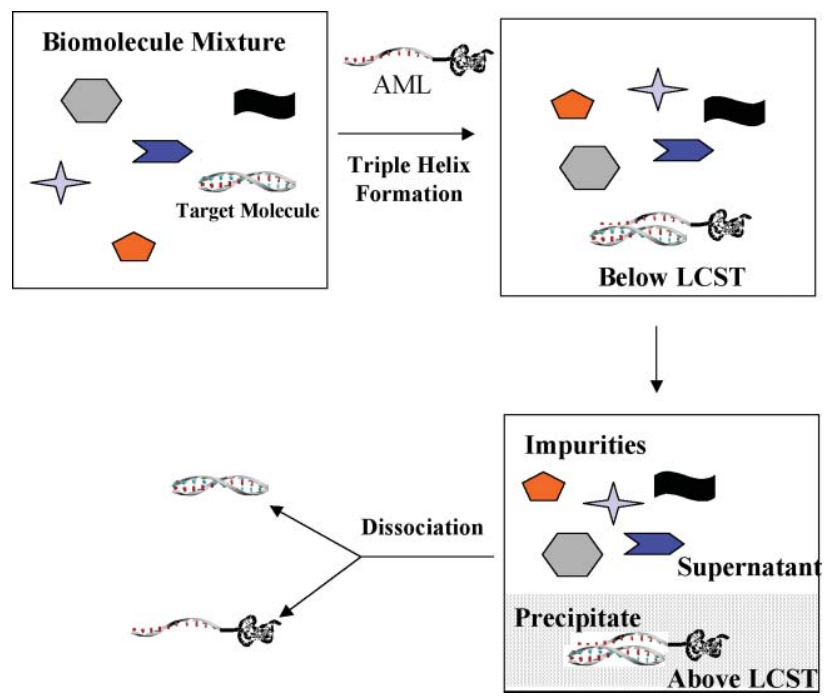

Fig. 4 Purification of pharmaceutical-grade plasmid DNA by triplex-helix affinity precipitation procedure. LCST: Lower critical solution temperature. Reprinted with permission from Wiley Interscience (ref. 71).

and excellent safety profile. ${ }^{73,74}$ However, the dosage which has been used in gene therapy is high, ${ }^{75-77}$ and the current purification techniques will probably not meet the demands if these drugs are routinely administered in the future. The triple-helix affinity precipitation of plasmid DNA by DNA- $b$-PNIPAM conjugates could serve as a practical system to provide large amounts of pharmaceutical-grade plasmid DNA.

Besides the isolation of plasmid DNA, DNA- $b$-PNIPAM conjugates have been applied to the affinity precipitation and separation of DNA-binding proteins. ${ }^{70}$ For that purpose, PNIPAM terminally functionalized with a psoralene group was photochemically crosslinked with ds DNA to form a graft architecture. When this side chain polymer containing a ds DNA backbone was enzymatically ligated to a non-PNIPAM-modified DNA segment encoding the so-called TATA-box, the corresponding TATA-boxbinding protein could be selectively separated from a protein mixture by thermal affinity precipitation. In the future, this elegant approach might be extended to the detection of unknown DNA binding proteins such as transcription factors from cell lysates.

\section{DBCs used in sensitive DNA detection}

Sensitive DNA detection is important in the fields of gene analysis, tissue matching, and forensic applications. The key challenge is to develop a material that efficiently senses the presence of ss or ds DNA and converts it to a detectable signal, either electrochemically or by means of fluorescence. A first approach towards using DNA block copolymers as probes for DNA detection was undertaken by Haralambidis and co-workers. ${ }^{78}$ The rationalization behind the use of employing a block copolymer architecture was that the nucleic acid part is needed for molecular recognition, while the polymer block allows the incorporation of multiple labels along the backbone. A synthetically challenging method was developed for achieving the linkage between the ODN and the organic polymer segment, since the polyamide was attached to the base of the nucleotide at the $5^{\prime}$-end. ${ }^{78}$ The polyamide unit was synthesized employing standard Fmoc chemistry. This allowed the 
incorporation of several pyrenylated amino acid building blocks into the peptide segment. ${ }^{79}$ Significant excimer fluorescence from the DNA- $b$-polyamide was detected due to the close proximity of the chromophores. The multimerization of labels resulted in an increase of the emission intensity, proving the concept of a polylabel strategy. Hybridization of DNA- $b$-polyamide with complementary sequences doubled the luminescence intensity of the probe. This was possibly due to less pronounced stacking interactions of the pyrene residues with nucleobases in the ds DNA compared to the ss DNA. However, real DNA detection was not realized.

Instead of DNA diblock copolymers, we developed a triblock architecture for DNA detection. This novel structural concept is based on fluorescence dequenching upon hybridization (Fig. 5A). The so-called "twin probe" consists of a central fluorene derivative as fluorophore to which two identical oligonucleotides are covalently attached. ${ }^{80}$ This probe architecture was applied in a homogenous hybridization assay with subsequent fluorescence spectroscopic analysis. The bioorganic hybrid structure was well-suited for sequence specific DNA detection, and even single-nucleotide polymorphisms (SNPs) were identified with high efficiency. The covalent attachment of two single-stranded oligonucleotides leads to strong quenching of the central fluorescence dye induced by the nucleobases, whereas when one oligonucleotide is coupled to the fluorophore no dequenching upon hybridization occurs. The twin

$\mathbf{A}$
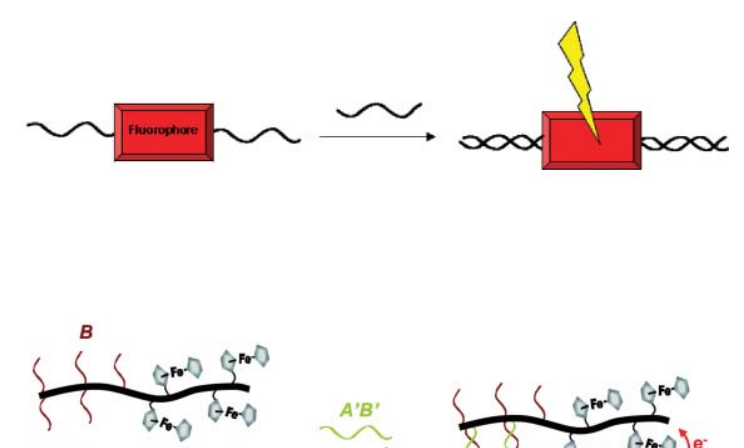

B

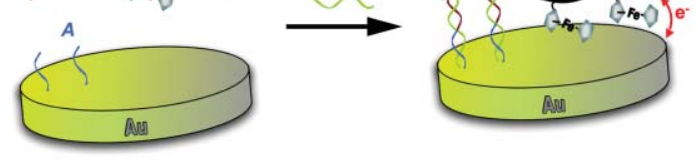

C

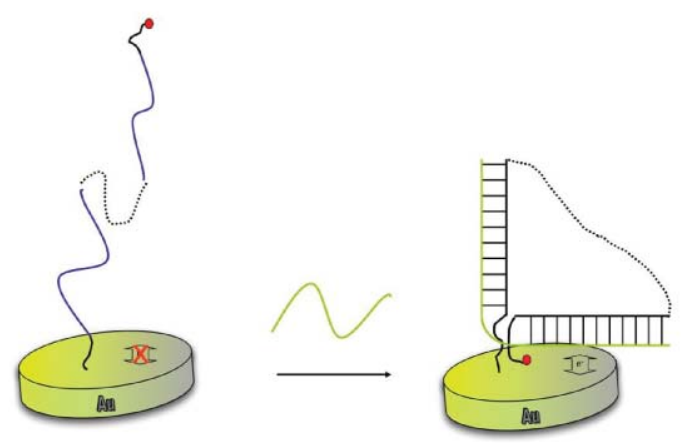

Fig. 5 DNA block copolymers used in sensitive DNA detection. A) The twin probe is applied for DNA detection by means of fluorescence in a homogenous hybridization essay (adapted from ref. 80). B) Electrochemical detection of DNA by graft architecture (adapted from ref. 45). C) Triblock architecture for the reagentless DNA detection (adapted from ref. 40). probe is characterized by supramolecular aggregate formation accompanied by red-shifted emission and broad fluorescence spectra. In the future, the central emitter unit will be extended to oligomeric conjugated materials with the aim of increasing the sensitivity of the probe.

In contrast to linear structures, a graft architecture for sensitive DNA detection was realized by Mirkin and co-workers, who reported the electrochemical detection of DNA by polynorborneneDNA hybrids (Fig. 5B). ${ }^{44,45}$ Two kinds of DBCs with either ferrocenyl or dibromoferrocenyl groups as well as ODNs were prepared by ring-opening metathesis polymerization (ROMP). With these DBCs, target concentrations as low as $100 \mathrm{pM}$ could be detected, which is one order of magnitude more sensitive than the previously reported system based on ferrocene-containing oligonucleotides. ${ }^{81}$

A structural alternative to graft architectures of DBCs used for electrochemical DNA detection is a linear topology (Fig. 5C). Grinstaff et al. prepared an A-B-A type triblock copolymer containing two DNA strands linked via a small, flexible PEO linker. ${ }^{40}$ The capture strand was functionalized with a terminal thiol for immobilization on a gold electrode. The probe strand contained a 5'-terminal redox-active reporter group, ferrocene. Upon binding of the target strand to the immobilized capture strand the distance between the 5'-terminal ferrocene and the electrode surface was decreased, resulting in an electrochemical signal. This DNA triblock copolymer gives rise to a sensitive reagentless electrochemical assay which is ideally suited for the continuous, rather than batch, monitoring of a flow of analyte. ${ }^{82}$ Compared to the above described graft architecture, the estimated detection limit of the assay was 200 pM of DNA.

\section{DBCs in nanoscience}

Nanotechnology has been one of the fastest developing research areas in recent years. One of the key objectives in this fascinating multidisciplinary field are nanoparticles, which most commonly exhibit sizes in the range of $10-100 \mathrm{~nm}$ and size-dependent properties different from the bulk materials. These objects can either be composed of inorganic ${ }^{83-85}$ or organic materials. ${ }^{86}$ Synthetic chemists have been extremely creative in finding new methods for the preparation of nanoparticles. The chemical synthesis techniques can, in principle, be divided into two general strategies: 1) the mechanical milling of raw material down to nanosized particles and 2) the conversion of the products or educts dissolved in suitable solvents into nanodispersed systems by precipitation, condensation or chemical synthesis. Especially, within the chemical routes towards nanoparticles, polymers are often involved, if one considers the preparation of polymer dispersions ${ }^{87-89}$ and dendrimers ${ }^{90,91}$ or the aggregation of block polymers. ${ }^{22,92}$ When the solvent environment of a linear block copolymer system is a selective solvent for one of the segments and the other polymer unit is insoluble, spherical micelles of nearly uniform size and shape are typically obtained, which can be regarded as nanoparticle systems. ${ }^{93}$

Translated into the context of amphiphilic DNA block copolymers, this means that nanoparticles containing a hydrophobic polymeric core and a ss DNA corona are obtained. In a previous paragraph, the advantages of such systems containing a hydrophobic core of PLGA and a shell of ss nucleic acids have been 
discussed in regard to delivery of antisense ODNs. However, amphiphilic DBC systems with polystyrene (PS) ${ }^{39}$ and poly(propylene oxide) (PPO) ${ }^{42,94}$ have also been synthesized.

The organic segment of DNA- $b$-PS polymers exhibited an $M_{n}$ of $5600 \mathrm{~g} \mathrm{~mol}^{-1}$ while the lengths of the ODNs were adjusted to be a 5mer, a 10-mer and a 25-mer. The diameter of the resulting micelles was measured by AFM and DLS, which are important tools for the characterization of superstructures formed from amphiphilic DBCs. The different lengths of the DNA segments resulted in tailorable diameters of the micelles ranging from $8-30 \mathrm{~nm}$. The AFM measurements, carried out in tapping mode on a mica surface in air, were consistent with the DLS data. These well-defined block copolymer micelles were employed to build up sequencespecific aggregates with DNA-modified gold nanoparticles. The aggregates could be reversibly disassembled by heating them above the melting temperature of the double-stranded DNA. This result paves the way to higher-ordered nanostructures defined by the recognition properties of DNA and the hydrophobic-hydrophobic interactions of the water insoluble polymer segments. In such a fashion, hybrid structures consisting of three classes of materialsorganic polymers, biological entities and inorganic moietieswere realized. We believe that in the future various functions will evolve from these materials, especially when the organic polymer bears additional features like luminescent or semiconducting properties.

The amphiphilic DBCs mentioned so far contain a hydrophobic block with high glass transition temperature $\left(T_{\mathrm{G}}\right)$, thus hindering the easy dissolution of the amphiphilic structures and investigation of the micellar properties because the so-called "frozen" micelles hardly reach a state of thermodynamic equilibrium. ${ }^{22}$ To overcome these drawbacks, our group has prepared DNA- $b$-PPO polymers in which the synthetic polymer block exhibits a low $T_{\mathrm{G}}\left(-70^{\circ} \mathrm{C}\right)$, which allows easy preparation of the micelle aggregates by just dissolving the DBCs in an aqueous medium. ${ }^{42}$ Moreover, the organic PPO segment has proven to be biocompatible with different cell types, which might be of importance if such structures are employed in living systems. ${ }^{95}$ The DNA- $b$-PPO formed spherical micelles, which were characterized in detail by AFM and DLS. In contrast to the work mentioned above, the AFM visualization was conducted in buffer by soft-tapping mode. The height of the micelles ranged from 6 to $18 \mathrm{~nm}$, which was consistent with the DLS measurements. These nanoparticles were introduced as a novel template for DNA-templated synthesis (DTS).

DTS has emerged as a tool for nucleic acid sensing, smallmolecule discovery, and reaction discovery with the help of translation, selection, and amplification methods previously available only to biomacromolecules like nucleic acids and proteins. ${ }^{96}$ Based on the effective molarity approach, complex small molecules and polymer products were generated employing multistep DTS.

As templates, ss DNA strands representing one-dimensional objects are commonly employed to assemble the reactant DNA. However, there is also one report in which a twodimensional template, a trisoligonucleotide, was used. ${ }^{97}$ We expanded the scope of DTS to three-dimensional templates using spherical aggregates of DNA- $b$-PPO polymers as a platform to run sequence-specific organic transformations. ${ }^{42}$ Several organic reactions proceeded in a programmable manner either at the corona of the micelles or at the interface between the biological and organic polymer blocks (Fig. 6). The yields of the reactions (isoindole formation, Michael addition and amide bond formation) were equivalent or better than for existing templates. Furthermore, hydrophobic reactants can accumulate within the core to produce higher yields. These templates composed of amphiphilic DBCs are of great importance for DNA-templated chemistry because it might allow sequence-specific programmable reactions to occur while being protected from the environment, as in a cellular system.

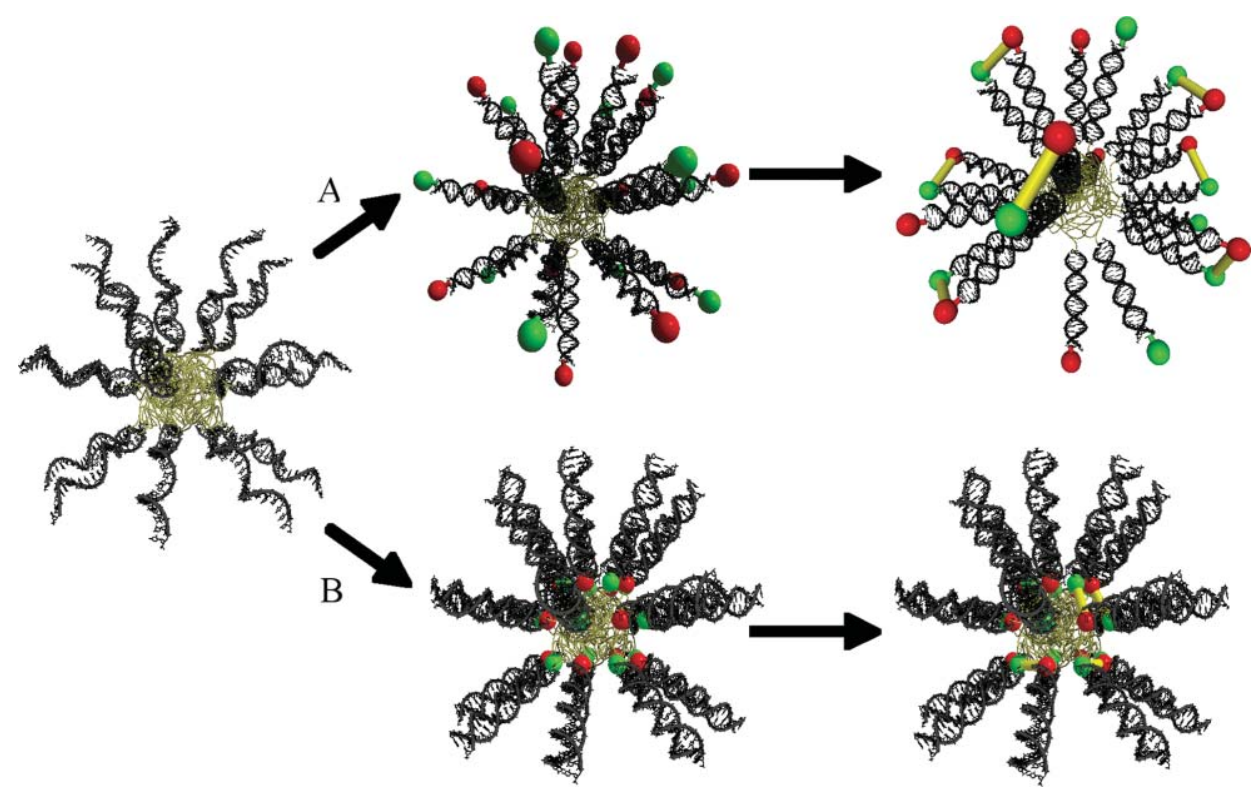

Fig. 6 Schematic representation of DNA-templated synthesis applying DNA block copolymers. The micelles resulting from these polymeric architectures consist of a hydrophobic core and a shell of DNA. Single-stranded micelles can be either hybridized with oligonucleotides which are equipped with reactants at the 5'- and 3'-ends. The subsequent chemical reaction proceeds at the rim of the micelle (A) or at the hydrophobic/hydrophilic interface (B), respectively (ref. 42). 
Besides spherical morphologies, amphiphilic block copolymers self-assemble into a large number of different supramolecular aggregates of various shapes which have recently been reported and pioneered by Eisenberg ${ }^{98-100}$ as well as by other research groups. ${ }^{101,102}$ These morphologies are important because of their specific applications in controlled drug delivery, encapsulating agents for catalysis, emulsifying agents, and in separation systems. ${ }^{103}$ The micelle sizes and shapes can be manipulated by the chemical nature of the polymer ${ }^{104}$ or by changing environmental conditions like salinity, $\mathrm{pH}$ and solvent composition. ${ }^{105}$ However, it is still a challenge to perfectly control the morphologies and dimensions of these aggregates. In our group, this aim was approached by employing DBCs and exploiting the self-recognition properties of the nucleic acid segments. DNA- $b$-PPO micelle morphologies were altered by hybridization, transforming the ss nucleic acid shell into ds DNA. ${ }^{94}$ While hybridization of DNA block copolymer aggregates with complementary short DNA has no significant impact on the structural properties, base pairing with long DNA templates induced a transformation from spherical into rod-like micelles (Fig. 7). The Watson-Crick motif aligned the hydrophobic polymer segments along the DNA double helix, which resulted in selective dimer formation. The length of the resulting rod-like micelles could even be adjusted precisely by varying the number of nucleotides in the templates. Salient features of this novel strategy are the sequence specificity and the structural uniformity of the resulting micelle aggregates. This study, for the first time, demonstrated that DNA nanostructures, which are usually generated using base pairing of complementary ss ODN sequences, ${ }^{17,18,106-110}$ can be built up by employing hydrophobic interactions, adding a new tool to the field of DNA nanotechnology with respect to structure formation.

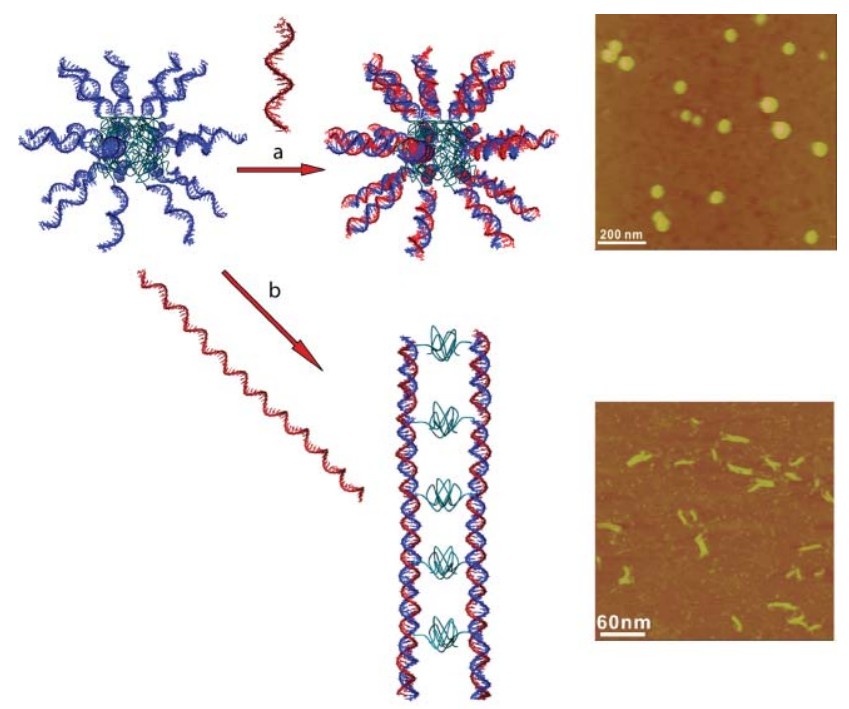

Fig. 7 Schematic representation of hybridization of ss DNA- $b$-PPO micelles with different DNA molecules. (a) Base pairing with a short complementary sequence yields micelles with a ds corona almost maintaining the structural properties of the ss aggregates. (b) Hybridization with long DNA templates results in rod-like micelles consisting of two parallel aligned double helices (ref. 94).

\section{DNA hydrogels based on DBCs}

In general, hydrogels are defined as crosslinked polymer networks. Two different network architectures containing DNA are known. The first class of DNA hydrogels was built up by chemically crosslinking ds DNA strands. ${ }^{111}$ As a crosslinking agent, ethylene glycol diglcidyl ether was employed. Such DNA gels showed a discontinuous volume transition when acetone was added to the network that was swollen in aqueous medium. At a concentration of $63 \%$ acetone, the volume of the gel fell by a factor of 15 , and the process was proven to be reversible. Such phase transitions are one reason why polymer networks have attracted the attention of many researchers. Recently, several groups have investigated synthetic polymer hydrogels and tried to induce phase transitions by external stimuli. ${ }^{112}$ Gels can expand or contract when triggered by tiny changes in temperature, light, solvent composition, or when target molecules are bound. The ability of the gels to undergo huge but reversible changes in volume allows unique new systems to be created mainly for the purpose of encapsulating and releasing materials. Since the synthetic polymer chains of the gels cannot bind with the target molecules selectively, conjugates of the receptors and the chain are needed. In contrast, DNA has inherently a unique chain structure able to bind with specific bio- and synthetic molecules. ${ }^{111,113}$ At this point, the second class of DNA networks is introduced. The characteristic of these structures is that the crossing points, not the polymer network, consist of DNA. Nagahara and co-workers prepared two different kinds of DBCs. Poly( $N, N$-dimethylacrylamide-co$N$-acryloyloxysuccinimide) was reacted with either an aminoterminated 10-mer ODN exclusively containing adenine (oligoA) or thymine bases (oligoT) to form graft architectures. ${ }^{46}$ A first hydrogel was realized by hybridizing the side chain polymer carrying oligoA with the conjugate containing oligoT. In a second route, a hydrogel was formed by hybridizing two oligoT-derivatized copolymers with a 20 -mer adenine crosslinking strand. Nagahara et al. prepared films of these hydrogel materials and characterized the hybridization behaviour by UV-monitored melting curves. The material exhibits two important properties. First, gel formation is reversible and the temperature of dissociation can be controlled by the composition and length of the ODN. Second, during the gelation process, which can be carried out at room temperature, the target molecule remains intact because of the mild and selective hydrogen bond formation between complementary DNA strands. Release of the target molecule might be achieved by denaturing the double-stranded DNA crosslinks. Inspired by this approach, Langrana $e t$ al. prepared DNA gels by adding a crosslinking strand to a mixture of two DNA-polyacrylamide graft architectures. ${ }^{48,49}$ This DNA sequence was designed in such a fashion that it was complementary to both ODNs of the DNA graft polymers. As described for the previous example, hydrogel formation was thermoreversible. But it was also possible to dissociate the DNA crosslinks without a thermal stimulus. This was achieved by introducing a toehold at the crosslinking strand or so-called fuel strand consisting of an additional ss DNA segment that does not hybridize with the DNA sidechains attached to the polyacrylamide. When a so-called removal strand that is a full complement to the fuel strand was added, the crosslinks could be efficiently disintegrated. This change in the degree of crosslinking was accompanied by a switch of the mechanical properties of the 
hydrogel. It needs to be pointed out here that all environmental parameters such as temperature and buffer conditions remained constant, so just the addition of a DNA strand induced a change in the stiffness of the network. These kind of sequence-responsive materials with modifiable bulk properties might be promising candidates for biotechnology applications.

\section{Outlook and perspectives}

The combination of DNA and synthetic polymers in a covalent fashion leads to engineered material properties of the hybrids that cannot be realized with the polymer or the nucleic acid as single entities. Several synthetic routes and coupling strategies are now available to produce ss DNA di- and triblock architectures. These methods especially allow one to vary the nature of the organic polymer to exhibit hydrophilic and hydrophobic properties, as well as thermoresponsive characteristics. However, a synthetic limitation remains regarding the nucleic acid segments. So far, the length of the DNA blocks is limited to around 40 nucleotides, which is rather small in comparison to naturally occurring nucleic acids like genomic or plasmid DNA. In the future, new synthetic strategies need to be explored that allow researchers to overcome this restriction. This could possibly be achieved by combining polymer synthetic methods with procedures that are applied in molecular biology. Since ss DNA block copolymers have been prepared almost exclusively up to now, another remaining synthetic task is the preparation of DNA multiblock structures that might be realized by hybridization of already existing ss DNA block copolymer building blocks.

DBCs have found promising applications in the field of antisense and antigene delivery, DNA detection, and in nanoscience. In the future more efforts must be devoted to optimize these applications and broaden their use in the fields of bio- and nanotechnology. With the use of DBCs in the context of nucleic acid delivery, a promising research direction, namely investigating the interaction of these materials with living cells, has been started. We believe that these materials are highly promising in this regard, especially in exploring the interactions of nanoparticles with natural systems. Morphologies, sizes and surface charges can already be tailored in micelle systems consisting of amphiphilic DBCs. Now it is time to study uptake and transport properties of the systems in cells and through biological barriers in regard to their physical parameters. Once these relations are understood, one could even consider using DNA block copolymer micelles for drug delivery. The high modularity of this micelle platform with regard to functionalization by just "clicking in" different components by hybridization has already been demonstrated in the test tube. This multifunctionalization might allow the targeting and release of drugs in desired tissues with a precision not yet achieved.

\section{Acknowledgements}

This work, as part of the European Science Foundation EUROCORES Programme BIONICS, was supported by funds from the DFG and the EC Sixth Framework Programme. Additional funding was provided through the ERA-CHEMISRY programme and the DFG-Sonderforschungsbereich 625 "From Single Molecules to Nanostructured Materials". The help of Minseok Kwak and
Roland Bauer for creating graphical representations is gratefully acknowledged.

\section{References}

1 P. Y. W. Dankers, M. C. Harmsen, L. A. Brouwer, M. J. A. Van Luyn and E. W. Meijer, Nat. Mater., 2005, 4, 568-574.

2 T. Kokubo, H. M. Kim and M. Kawashita, Biomaterials, 2003, 24, 2161-2175.

3 V. Schreiber, F. Dantzer, J. C. Ame and G. de Murcia, Nat. Rev. Mol. Cell Biol., 2006, 7, 517-528.

4 G. Krampitz and G. Graser, Angew. Chem., Int. Ed. Engl., 1988, 27, $1145-1156$.

5 L. Addadi and S. Weiner, Angew. Chem., Int. Ed. Engl., 1992, 31, 153169.

6 G. L. Conn, D. E. Draper, E. E. Lattman and A. G. Gittis, Science, 1999, 284, 1171-1174.

7 P. Nissen, M. Kjeldgaard, S. Thirup, G. Polekhina, L. Reshetnikova, B. F. C. Clark and J. Nyborg, Science, 1995, 270, 1464-1472.

8 S. J. Park, A. A. Lazarides, C. A. Mirkin, P. W. Brazis, C. R. Kannewurf and R. L. Letsinger, Angew. Chem., Int. Ed., 2000, 39, 3845-3848.

9 S. J. Park, T. A. Taton and C. A. Mirkin, Science, 2002, 295, 15031506.

10 T. A. Taton, C. A. Mirkin and R. L. Letsinger, Science, 2000, 289, $1757-1760$

11 E. Braun, Y. Eichen, U. Sivan and G. Ben-Yoseph, Nature, 1998, 391, $775-778$.

12 K. Keren, R. S. Berman, E. Buchstab, U. Sivan and E. Braun, Science, 2003, 302, 1380-1382.

13 C. D. Mao, W. Q. Sun, Z. Y. Shen and N. C. Seeman, Nature, 1999, 397, 144-146.

14 M. H. Caruthers, Science, 1985, 230, 281-285.

15 J. D. Watson and F. H. C. Crick, Nature, 1953, 171, 737-738.

16 J. D. Watson and F. H. C. Crick, Nature, 1953, 171, 964-967.

17 P. W. K. Rothemund, Nature, 2006, 440, 297-302.

18 N. C. Seeman, Nature, 2003, 421, 427-431.

19 C. X. Lin, Y. Liu, S. Rinker and H. Yan, ChemPhysChem, 2006, 7, $1641-1647$.

20 M. K. Beissenhirtz and I. Willner, Org. Biomol. Chem., 2006, 4, $3392-$ 3401.

21 I. W. Hamley, The physics of block copolymers, Oxford University Press, Oxford, 1998.

22 S. Forster, V. Abetz and A. H. E. Muller, Adv. Polym. Sci., 2004, 166, $173-210$.

23 T. K. Bronich, H. K. Nguyen, A. Eisenberg and A. V. Kabanov, J. Am. Chem. Soc., 2000, 122, 8339-8343.

24 M. Thomas and A. M. Klibanov, Proc. Natl. Acad. Sci. U. S. A., 2002, 99, 14640-14645.

25 J. H. Jeong, S. H. Kim, S. W. Kim and T. G. Park, Bioconjugate Chem., $2005,16,1034-1037$

26 Y. G. Takei, T. Aoki, K. Sanui, N. Ogata, T. Okano and Y. Sakurai, Bioconjugate Chem., 1993, 4, 42-46.

27 Y. G. Takei, M. Matsukata, T. Aoki, K. Sanui, N. Ogata, A. Kikuchi, Y. Sakurai and T. Okano, Bioconjugate Chem., 1994, 5, 577-582.

28 R. B. Fong, Z. L. Ding, C. J. Long, A. S. Hoffman and P. S. Stayton, Bioconjugate Chem., 1999, 10, 720-725.

29 M. Oishi, T. Hayama, Y. Akiyama, S. Takae, A. Harada, Y. Yarnasaki, F. Nagatsugi, S. Sasaki, Y. Nagasaki and K. Kataoka, Biomacromolecules, 2005, 6, 2449-2454.

30 J. H. Jeong, S. W. Kim and T. G. Park, J. Controlled Release, 2003, 93, $183-191$.

31 D. Umeno, T. Mori and M. Maeda, Chem. Commun., 1998, 14331434.

32 D. Umeno, M. Kawasaki and M. Maeda, Bioconjugate Chem., 1998, 9, 719-724.

33 M. Oishi, S. Sasaki, Y. Nagasaki and K. Kataoka, Biomacromolecules, 2003, 4, 1426-1432.

34 M. Murata, W. Kaku, T. Anada, Y. Sato, T. Kano, M. Maeda and Y. Katayama, Bioorg. Med. Chem. Lett., 2003, 13, 3967-3970.

35 J. H. Jeong, S. H. Kim, S. W. Kim and T. G. Park, J. Biomater. Sci., Polym. Ed., 2005, 16, 1409-1419.

36 S. Cogoi, M. Ballico, G. M. Bonora and L. E. Xodo, Cancer Gene Ther., 2004, 11, 465-476. 
37 J. Sanchez-Quesada, A. Saghatelian, S. Cheley, H. Bayley and M. R. Ghadiri, Angew. Chem., Int. Ed., 2004, 43, 3063-3067.

38 M. Oishi, F. Nagatsugi, S. Sasaki, Y. Nagasaki and K. Kataoka, ChemBio Chem, 2005, 6, 718-725.

39 Z. Li, Y. Zhang, P. Fullhart and C. A. Mirkin, Nano Lett., 2004, 4, $1055-1058$.

40 C. E. Immoos, S. J. Lee and M. W. Grinstaff, J. Am. Chem. Soc., 2004, 126, 10814-10815.

41 B. de Lambert, C. Chaix, M. T. Charreyre, A. Laurent, A. Aigoui, A. Perrin-Rubens and C. Pichot, Bioconjugate Chem., 2005, 16, 265274.

42 F. E. Alemdaroglu, K. Ding, R. Berger and A. Herrmann, Angew. Chem., Int. Ed., 2006, 45, 4206-4210.

43 C. Minard-Basquin, C. Chaix, C. Pichot and B. Mandrand, Bioconjugate Chem., 2000, 11, 795-804.

44 K. J. Watson, S. J. Park, J. H. Im, S. T. Nguyen and C. A. Mirkin, J. Am. Chem. Soc., 2001, 123, 5592-5593.

45 J. M. Gibbs, S. J. Park, D. R. Anderson, K. J. Watson, C. A. Mirkin and S. T. Nguyen, J. Am. Chem. Soc., 2005, 127, 1170-1178.

46 S. Nagahara and T. Matsuda, Polym Gels Networks, 1996, 4, 111-127.

47 C. Minard-Basquin, C. Chaix and C. Pichot, Nucleosides, Nucleotides Nucleic Acids, 2001, 20, 895-899.

48 D. C. Lin, B. Yurke and N. A. Langrana, J. Biomech. Eng., 2004, 126, 104-110.

49 D. C. Lin, B. Yurke and N. A. Langrana, J. Mater. Res., 2005, 20, 1456-1464.

50 C. A. Stein and Y. C. Cheng, Science, 1993, 261, 1004-1012.

51 J. B. Opalinska and A. M. Gewirtz, Nat. Rev. Drug Discovery, 2002, 1, 503-514.

52 J. H. Jeong and T. G. Park, Bioconjugate Chem., 2001, 12, 917-923.

53 J. H. Jeong, S. W. Kim and T. G. Park, Bioconjugate Chem., 2003, 14, 473-479.

54 C. Morassutti, B. Scaggiante, L. E. Xodo, B. Dapas, G. Paroni, G. Tolazzi and F. Quadrifoglio, Antisense Nucleic Acid Drug Dev., 1999, 9, 261-270.

55 S. Cogoi, C. Suraci, E. Del Terra, S. Diviacco, G. Van der Marel, J. Van Boom, F. Quadrifoglio and L. Xodo, Antisense Nucleic A, 2000, 10, 283-295.

56 S. Cogoi, V. Rapozzi, F. Quadrifoglio and L. Xodo, Biochemistry, 2001, 40, 1135-1143.

57 V. Rapozzi, S. Cogoi, P. Spessotto, A. Risso, G. M. Bonora, F. Quadrifoglio and L. E. Xodo, Biochemistry, 2002, 41, 502-510.

58 J. Joseph, J. C. Kandala, D. Veerapanane, K. T. Weber and R. V. Guntaka, Nucleic Acids Res., 1997, 25, 2182-2188.

59 E. M. McGuffie, D. Pacheco, G. M. R. Carbone and C. V. Catapano, Cancer Res., 2000, 60, 3790-3799.

60 F. X. Barre, S. Ait-Si-Ali, C. Giovannangeli, R. Luis, P. Robin, L. L. Pritchard, C. Helene and A. Harel-Bellan, Proc. Natl. Acad. Sci. U. S. A., 2000, 97, 3084-3088.

61 A. M. Stutz, J. Hoeck, F. Natt, B. Cuenoud and M. Woisetschlager, J. Biol. Chem., 2001, 276, 11759-11765.

62 F. Rininsland, T. R. Johnson, C. L. Chernicky, E. Schulze, P. Burfeind, J. Ilan and J. Ilan, Proc. Natl. Acad. Sci. U. S. A., 1997, 94, 5854-5859.

63 G. M. Bonora, E. Ivanova, V. Zarytova, B. Burcovich and F. M. Veronese, Bioconjugate Chem., 1997, 8, 793-797.

64 M. Ballico, S. Drioli, F. Morvan, L. Xodo and G. M. Bonora, Bioconjugate Chem., 2001, 12, 719-725.

65 M. Heskins and J. E. Guillet, J. Macromol. Sci., Pure Appl. Chem., 1968, 2, 1441-1455.

66 Y. H. Bae, T. Okano, R. Hsu and S. W. Kim, Makromol. Chem., Rapid Commun., 1987, 8, 481-485.

67 Y. G. Takei, T. Aoki, K. Sanui, N. Ogata, T. Okano and Y. Sakurai, Bioconjugate Chem., 1993, 4, 341-346.

68 D. Umeno and M. Maeda, Chem. Lett., 1999, 381-382.

69 T. Mori, D. Umeno and M. Maeda, Biotechnol. Bioeng., 2001, 72, 261-268.

70 N. Soh, D. Umeno, Z. L. Tang, M. Murata and M. Maeda, Anal. Sci., 2002, 18, 1295-1299.

71 M. D. Costioli, I. Fisch, F. Garret-Flaudy, F. Hilbrig and R. Freitag, Biotechnol. Bioeng., 2003, 81, 535-545.
72 M. Murata, W. Kaku, T. Anada, N. Soh, Y. Katayama and M. Maeda, Chem. Lett., 2003, 32, 266-267.

73 A. Mountain, Trends Biotechnol., 2000, 18, 119-128.

74 F. Berger, C. Canova, A. L. Benabid and D. Wion, Nat. Biotechnol., $1999,17,517-517$.

75 D. Robertson, Nat. Biotechnol., 2001, 19, 604-605.

76 E. Sheu, S. Rothman, M. German, X. G. Wang, M. Finer and B. M. McMahon, Curr. Opin. Mol. Ther., 2003, 5, 420-427.

77 H. Aihara and J. Miyazaki, Nat. Biotechnol., 1998, 16, 867-870.

78 G. Tong, J. M. Lawlor, G. W. Tregear and J. Haralambidis, J. Org. Chem., 1993, 58, 2223-2231.

79 G. Tong, J. M. Lawlor, G. W. Tregear and J. Haralambidis, J. Am. Chem. Soc., 1995, 117, 12151-12158.

80 E. Ergen, M. Weber, J. Jacob, A. Herrmann and K. Mullen, Chem.Eur. J., 2006, 12, 3707-3713.

81 C. J. Yu, Y. J. Wan, H. Yowanto, J. Li, C. L. Tao, M. D. James, C. L. Tan, G. F. Blackburn and T. J. Meade, J. Am. Chem. Soc., 2001, 123, 11155-11161.

82 C. Fan, K. W. Plaxco and A. J. Heeger, Proc. Natl. Acad. Sci. U. S. A., 2003, 100, 9134-9137.

83 V. Lindberg and B. Hellsing, J. Phys.: Condens. Matter, 2005, 17, S1075-S1094.

84 T. Trindade, P. O'Brien and N. L. Pickett, Chem. Mater., 2001, 13, 3843-3858.

85 A. Eychmuller, J. Phys. Chem. B, 2000, 104, 6514-6528.

86 D. Horn and J. Rieger, Angew. Chem., Int. Ed., 2001, 40, 4331-4361.

87 D. Distler, Waessrige Polymerdispersionen: Synthese, Eigenschaften, Anwendungen, Wiley-VCH, Weinheim/Chichester, 1999.

88 J. M. Asua, Polymeric dispersions: Principles and applications, Kluwer Academic, Dordrecht/London, 1997.

89 T. Kietzke, D. Neher, K. Landfester, R. Montenegro, R. Guntner and U. Scherf, Nat. Mater., 2003, 2, 408-412.

90 C. Zhisheng and S. L. Cooper, Adv. Mater., 2000, 12, 843.

91 G. R. Newkome, C. N. Moorefield and F. Voegtle, Dendrimers and dendrons: concepts, syntheses, applications, Wiley-VCH, Weinheim/Chichester, 2001.

92 I. W. Hamley, Nanotechnology, 2003, 14, R39-R54.

93 S. Forster and M. Antonietti, Adv. Mater., 1998, 10, 195-217.

94 K. Ding, F. E. Alemdaroglu, M. Börsch, R. Berger and A. Herrmann, Angew. Chem., Int. Ed., 2007, 46, 1172-1175.

95 D. W. Miller, E. V. Batrakova, T. O. Waltner, V. Y. Alakhov and A. V. Kabanov, Bioconjugate Chem., 1997, 8, 649-657.

96 X. Y. Li and D. R. Liu, Angew. Chem., Int. Ed., 2004, 43, 4848-4870.

97 L. H. Eckardt, K. Naumann, W. M. Pankau, M. Rein, M. Schweitzer, N. Windhab and G. von Kiedrowski, Nature, 2002, 420, 286-286.

98 I. Astafieva, X. F. Zhong and A. Eisenberg, Macromolecules, 1993, 26, 7339-7352.

99 M. Moffitt, K. Khougaz and A. Eisenberg, Acc. Chem. Res., 1996, 29, 95-102.

100 D. E. Discher and A. Eisenberg, Science, 2002, 297, 967-973.

101 Y. Kakizawa and K. Kataoka, Adv. Drug Delivery Rev., 2002, 54, 203-222.

102 G. W. M. Vandermeulen and H. A. Klok, Macromol. Biosci., 2004, 4, 383-398.

103 P. Alexandridis and B. Lindman, Amphiphilic Block Copolymers: SelfAssembly and Applications, Elsevier, New York, 2000.

104 S. Jain and F. S. Bates, Science, 2003, 300, 460-464.

105 Y. S. Yu, L. F. Zhang and A. Eisenberg, Macromolecules, 1998, 31, 1144-1154.

106 N. C. Seeman, Angew. Chem., Int. Ed., 1998, 37, 3220-3238.

107 J. Wengel, Org. Biomol. Chem., 2004, 2, 277-280.

108 K. V. Gothelf and T. H. LaBean, Org. Biomol. Chem., 2005, 3, $4023-$ 4037.

109 U. Feldkamp and C. M. Niemeyer, Angew. Chem., Int. Ed., 2006, 45, 1856-1876.

110 A. Condon, Nat. Rev. Genet., 2006, 7, 565-575.

111 T. Amiya and T. Tanaka, Macromolecules, 1987, 20, 1162-1164.

112 J. Elisseeff, A. Ferran, S. Hwang, S. Varghese and Z. Zhang, Stem Cells Dev., 2006, 15, 295-303.

113 E. Takushi, Thermochim. Acta, 1998, 308, 75-76. 